4 A good network of support is always of importance, particularly outside of work, and I tend to gravitate towards friends working in othr professions to 'switch off' from the stress dentistry can bring on. My non-dentist friends are on hand to remind me life isn't just about teeth!

My other interests outside of work include taking part in voluntary opportunities including serving and preparing community meals and gate-stewarding for music festivals. I also enjoy keeping active: I am a black belt in Jujitsu which is something I achieved alongside my brothers growing up (whilst simultaneously showing them who's boss!).

Regarding my own oral health, I remember learning about the caries cycle and process as an undergraduate student and this is something that really resonated with me. Colleagues, friends and family who know me will all describe me as 'pedantic' in this respect as I am keen on finishing the majority of my meals with cheese. Fizzy drinks are a no-go in the house and I must admit I am a very avid flosser.
Throughout my career thus far as a DCT I have been provided with many opportunities to teach a variety of colleagues in various settings including: undergraduate dental students, foundation dentists, fellow DCTs and physician associates. Following this, I was enthused to enrol on a postgraduate certificate in 'Teaching and Learning in Clinical Practice' which I am currently undertaking. I hope that the theories and concepts I learn in these modules will be reflected in my practice. I am looking forward to my future career in the dental profession.

\title{
Facial surgeons call for better frontline staff training as lockdown may lead to increase in incidents
}

New research ${ }^{1}$ from oral and maxillofacial surgeons (OMFS) at King's College Hospital in South London has called for targeted training of frontline staff to improve recognition of domestic violence victims so appropriate safeguarding referral protocols are implemented.

The call from maxillofacial staff at King's follows the results of an 18-month study of all domestic violence cases with severe facial injuries treated by the King's OMFS team, and survey findings that looked at the attitudes of the maxillofacial team to recognising domestic violence. The move comes at a time when existing studies have found facial injuries reported in $76 \%$ to $94 \%$ of domestic violence cases, and make up $32 \%$ of all violent crimes.

The survey revealed frontline maxillofacial staff who treated domestic violence victims in the emergency department (ED) were only 'somewhat confident' in recognising victims of abuse. The accompanying case review confirmed that the majority of victims had been assaulted by current or ex-partners, and punched in the face or assaulted with household objects such as a knife or lamp.

Senior author, Consultant Maxillofacial Surgeon Kathy Fan explained that because facial injuries are common in domestic violence cases, maxillofacial teams are ideally placed to screen for victims and alert safeguarding.

Co-author Holly Boyes (pictured), maxillofacial junior trainee who works

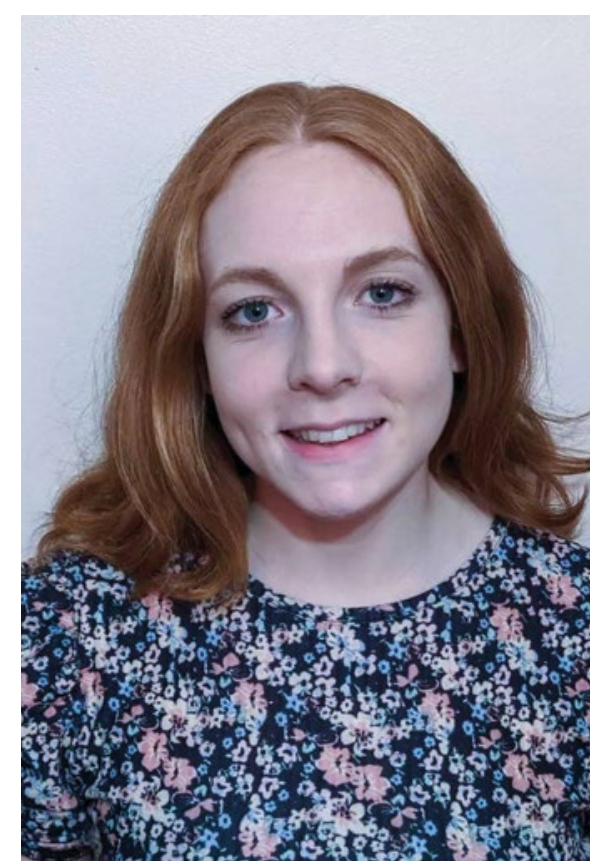

them and made the referral. It seemed like a bit of a relief to the patient.'

She admitted that she hadn't been comfortable about asking, and realised that her colleagues would probably be in a similar position. She took the survey findings to the monthly maxillofacial team meeting and invited the King's safeguarding team to do some training about how to go about asking those difficult questions.

'If it's happening at King's, then maxillofacial teams everywhere are missing the signs of domestic violence. We're in the right place to offer the support of safeguarding teams to protect patients in the short and long-term.'

Kathy Fan added: 'All healthcare staff play a part in supporting victims of domestic violence, and COVID-19 (coronavirus) self-isolation may lead to an increase in cases. But, don't forget, King's together with other NHS hospital safeguarding teams and Victim Support are still in place, and still working to help people.

'BAOMS is right to advocate for increased training, so that staff can feel confident asking and acting on signs of domestic abuse, particularly in difficult situations such as when children or a partner is present.'

\section{Reference}

1. Boyes H, Fan K. Maxillofacial injuries associated with domestic violence: experience at a major trauma centre. Br J Oral Maxillofac Surg 2020; 58: 185-189. 